\title{
Autoeficacia en la prevención sexual del Sida: la influencia del género
}

\author{
Rafael Ballester ${ }^{*}$, María Dolores Gil-Llario ${ }^{2}$, Estefanía Ruiz-Palomino ${ }^{1}$ y Cristina Giménez-García ${ }^{1}$ \\ ${ }^{1}$ Universitat Jaume I de Castellón, España \\ ${ }^{2}$ Universitat de València, España
}

\begin{abstract}
Resumen: La literatura científica apunta a que la escasa autoeficacia para el uso del preservativo explicaría muchas conductas de riesgo de transmisión del VIH en jóvenes. Se analiza la autoeficacia de los jóvenes en el uso del preservativo, la existencia de diferencias de género y la relación con variables relevantes en la prevención. Se aplicó la "Subescala de Autoeficacia en el Uso del Preservativo", incluida en el Cuestionario de Prevención del Sida (Ballester, Gil y Giménez, 2007), a 3540 universitarios (50.9\% mujeres, $49.1 \%$ hombres). Los resultados indican que los jóvenes obtienen puntuaciones medias-altas en autoeficacia, mostrándose la menor puntuación en el autocontrol en el momento de excitación. Se obtienen diferencias estadísticamente significativas en distintas situaciones según el género, siendo las mujeres las que se perciben más autoeficaces que los hombres, sobre todo en situaciones relacionadas con una posible evaluación negativa por parte de la pareja al sugerir su uso; y menos que ellos en aquellas referidas a ámbito público (comprar preservativos). Además, la autoeficacia correlacionó significativamente con la intención de conducta preventiva, la mayor frecuencia autoinformada de uso del preservativo y la percepción de eficacia del preservativo en la prevención del VIH. Las futuras intervenciones preventivas deberían incluir un componente de autoeficacia y una perspectiva de género.

Palabras clave: Autoeficacia; Uso preservativo; Jóvenes; Género; Preven-
\end{abstract} ción sida.

\section{Introducción}

Según el Programa de las Naciones Unidas para el Desarrollo (2006), el VIH ha infligido el "mayor revés individual en el desarrollo bumano" de la historia moderna. En el último informe epidemiológico de ONUSIDA se cifra en 33 millones las personas que viven con VIH en el mundo, siendo los jóvenes entre 15 y 24 años, el colectivo que representa la mitad de nuevas infecciones a escala mundial (UNAIDS, 2010). En el caso concreto de España, los jóvenes entre 15 y 34 años constituyen alrededor del $30 \%$ de todas las nuevas infecciones por el VIH (Secretaría del Plan Nacional sobre el Sida, 2009).

Desde la aparición del VIH, los esfuerzos preventivos por erradicar la expansión de la epidemia, se han centrado en proporcionar a los jóvenes información básica sobre los comportamientos sexuales de riesgo y la necesidad de prevenir la transmisión del VIH. Sin embargo, como ha demostrado la literatura científica, los conocimientos son un componente necesario pero no suficiente para lograr la realización de conductas sexuales seguras (Bayés, 1995). En general, algunos estudios realizados con muestras de estudiantes universitarios de diferentes comunidades autónomas de España demuestran que sigue existiendo un porcentaje significativo que no usa el preservativo de forma sistemática en sus

\section{* Dirección para correspondencia [Correspondence address]}

Rafael Ballester Arnal. Departamento de Psicología Básica, Clínica y Psicobiología. Facultad de Ciencias de la Salud. Universitat Jaume I. Avenida de Vicent Sos Baynat s/n, 12071 Castellón de la Plana (España), E-mail: rballest@psb.uji.es
Title: Self-efficacy in AIDS sexual prevention: influence of gender.

Abstract: The scientific literature suggests that a low self-efficacy for condom use may be related to many risky behaviors for HIV transmission in young people. We analyze the self-efficacy of condom use among youth, the existence of gender differences and the relationship with other relevant variables in HIV prevention. The "Self-Efficacy Condom Use Subscale", included in the AIDS Prevention Questionnaire (Ballester, Gil and Giménez, 2007), was administrated to 3540 university students $(50.9 \%$ women, $49.1 \%$ men). The results showed that the sample obtained medium-high scores on self-efficacy, showing the lowest scores when behavior involves their sexual control at the time of excitation. Statistically significant differences were obtained in different situations according to gender. In this regard, women perceived themselves higher condom use selfefficacy than men, especially when their suggestion about condom use might cause a negative evaluation to the partner; and unless than men those relating to public domain (buying condoms). Moreover, self-efficacy correlated significantly with preventive behavior intention, increased selfreported frequency of condom use and perceived effectiveness of condoms in HIV prevention. Future preventive interventions should include an element of self-efficacy and a differentiated perspective by gender.

Keywords: Self-efficacy; Condom use; Young; Gender; AIDS prevention.

relaciones sexuales (Ballester, Gil, Giménez y Ruiz, 2009; Lameiras, Faílde, Saco y Rodríguez, 2006; Planes, Gras, Soto y Font-Mayolas, 2000).

Este hecho ha motivado que desde diferentes modelos teóricos, se haya intentado proporcionar un marco de referencia más amplio, que nos acercara a la comprensión de los comportamientos sexuales de riesgo en población joven (Espada y Quiles, 2001). En este sentido, desde la perspectiva de la Teoría Social Cognitiva, Bandura (1994) propuso el Modelo de Autoeficacia como una explicación de las conductas sexuales de riesgo para la infección por VIH. Este autor entiende que la conducta está mediada por un proceso de evaluación cognitiva en el cual las personas integran conocimientos, expectativas de resultados, estados emocionales, influencias sociales y experiencias pasadas, de forma que crean una estimación de su habilidad para afrontar una determinada situación. Por lo tanto, que una persona utilice el preservativo, no sólo dependerá de los recursos que disponga para utilizarlo, sino también de la percepción de sí misma como capaz de controlar su propio comportamiento (Wulfert y Wan, 1993).

Algunos estudios realizados con población universitaria han señalado la importancia de la autoeficacia, no sólo como uno de los factores predictores de la intención del uso del preservativo (Mashegoane, Moalusi, Ngoepe y Peltzer, 2004) o de la utilización del mismo en las relaciones sexuales (Dilorio, Dudley, Soet, Watkins y Maibach, 2000; Ozakinci y Weinman, 2006; Svenson, Oestergren, Merlo y Rastam, 2002), sino también como una de las variables explicativas del mantenimiento del cambio tras la aplicación de una intervención preventiva (O’Leary, Jemmott y Jemmott III, 
2008) o de la adherencia al tratamiento antirretroviral en pacientes con VIH (Erlen, Cha, Kim, Caruthers y Sereika, 2010). Es por ello que un reciente metanálisis de aquellos elementos que la literatura especializada ha considerado relevantes en la prevención (Casey, Timmermann, Allen, Krahn y LaPlant, 2009), ha indicado la importancia de incluir y entrenar el componente de autoeficacia en el uso del preservativo en los programas educativos sobre VIH.

Sin embargo, a pesar de los trabajos que demuestran la relevancia de la autoeficacia en el uso del preservativo, todavía no existe un consenso claro sobre su conceptualización y evaluación en este ámbito. Su multidimensionalidad, por un lado, dificulta el diseño de instrumentos de evaluación que aglutinen la gran cantidad de aspectos necesarios para la realización de un comportamiento preventivo. Por otro, hace necesaria una aplicación específica del concepto a aquellas situaciones referidas a la utilización del preservativo en las relaciones sexuales incluyendo el tipo de pareja ( $\mathrm{Pla}-$ nes, 1995). En este sentido, observamos que cada autor acentúa aquellos aspectos que considera más relevantes en la realización de un comportamiento preventivo. Así, por ejemplo, Weeks, Levy, Zhu, Perhats, Handler y Flay (1995), identificaron dos tipos de autoeficacia: la de uso, como la habilidad de conseguir y utilizar las medidas preventivas (por ejemplo: comprar preservativos), y la de rechazo, como la habilidad necesaria para no dejarse inducir por situaciones que implican conductas de riesgo (por ejemplo: convencer a la pareja para utilizar el preservativo).

Si bien es cierto que evaluar diferentes situaciones relacionadas con el uso del preservativo enriquecerá el estudio de la autoeficacia en este ámbito, también lo es que el género puede ser un elemento a tener en cuenta a la hora de aplicar una intervención preventiva. La mayor parte de las investigaciones revisadas identifican la existencia de diferencias de género en la autoeficacia relacionada con el uso del preservativo. Sin embargo, mientras algunas encuentran puntuaciones más elevadas en las mujeres (Burns y Dillon, 2005; Parsons, Halkitis, Bimbi y Borkowski, 2000), otras lo hacen en los hombres (Farmer y Meston, 2006; Lindemann y Brigham, 2003). En el contexto sexual, la desigualdad en los roles de género emerge como factor relevante cuando consideramos la autoeficacia en el uso del preservativo. Por ejemplo, los estudiantes universitarios evaluados en el estudio de Quatrella (2000) atribuían la responsabilidad de conseguir o poner los preservativos a los hombres, y la de persuadir sobre su uso o comprobar que se ha utilizado correctamente a las mujeres. Como demuestran algunos estudios, todavía en la actualidad, existen estereotipos sexistas y percepciones tradicionales de la masculinidad, que dejan a la mujer en clara desventaja y vulnerabilidad, sobre todo en los países en desarrollo. En las sociedades occidentales, aunque las desigualdades de género son menos perceptibles, nos encontramos con expectativas sexistas, sobre cómo debe comportarse un hombre o una mujer en una relación sexual (Allen, Emmers-Sommer y Crowell, 2002; Matsuura, 2009; MúñozSilva, Sánchez-García, Martins y Nunes, 2009), que pueden dificultar la prevención frente al VIH. Por ello, el presente estudio tiene como objetivo, por un lado, evaluar el nivel de autoeficacia en el uso del preservativo en diferentes momentos de la relación sexual; por otro, averiguar si existen diferencias de género en una muestra de jóvenes universitarios; y en tercer lugar, indagar la relación existente entre autoeficacia en el uso del preservativo y diferentes aspectos relacionados con la prevención de la transmisión del VIH.

\section{Método}

\section{Participantes}

Se empleó una muestra de estudiantes de las distintas facultades de la Universitat Jaume I de Castellón, que acudieron a mesas divulgativas organizadas por la Unidad de Investigación sobre Sexualidad y Sida (UNISEXSIDA) con motivo del Día del Sida. De los 3530 participantes que constituían el total de la muestra, 1798 eran mujeres (50.9\%) y 1732 hombres (49.1\%). El rango de edades estaba comprendido entre 17 y 26 años $(M=20.72, D T=2.27)$. La mayor parte de la muestra evaluada afirmó tener relaciones sexuales en el momento de la evaluación (82.1\%).

\section{Instrumento}

Para recoger los datos se utilizó el Cuestionario de Prevención del Sida (CPS) de Ballester, Gil y Giménez (2007). Este instrumento está compuesto por un total de 65 ítems que intentan recoger aquellos componentes que en los distintos modelos de prevención sobre VIH se han considerado relevantes. Los datos de consistencia interna y fiabilidad testretest del instrumento son aceptables, obteniéndose un Alpha de Cronbach de .70 y una correlación de .83 entre el test y el retest. Obtuvo además una buena puntuación de validez concurrente, .79, con la Encuesta sobre Sida (Ballester et al., 2007).

De acuerdo con el objetivo del presente estudio, se ha utilizado el componente de autoeficacia, el cual está compuesto por 7 enunciados que describen diferentes habilidades relacionadas con el uso del preservativo en distintos momentos de una relación sexual (véase Figura 1): antes de la relación sexual, en la compra del preservativo, en la negociación con la pareja, a la hora de ponérselo, en una situación en la que se ha consumido alcohol u otras drogas y en una situación de máxima excitación sexual. Los enunciados fueron seleccionados tanto a partir de la literatura sobre el tema como del acuerdo interjueces entre cuatro expertos en la prevención del VIH y de embarazos no deseados. Los participantes deben responder en función de su grado de acuerdo mediante un formato tipo Likert con 6 alternativas. A pesar que los ítems están formulados tanto en sentido directo (ítems 1, 2, 5 y 7) como en sentido inverso (ítems 3, 4 y 6), es importante saber que para la presentación de los resultados, hemos transformado los valores de forma que, en todos los enunciados, mayor puntuación signifique mayor percep- 
ción de autoeficacia. Además de las puntuaciones en cada ítem, se obtiene una puntuación total resultado de la suma de todas las puntuaciones siendo el máximo de 35 puntos. Las propiedades psicométricas de la presente escala fueron evaluadas con una muestra de 4503 estudiantes universitarios, obteniendo un Alpha de Cronbach de .52 (Gómez, Ba- llester, Gil, Gil y Salmerón, 2009). El análisis de la fiabilidad para la muestra del presente estudio es de .542. Ambas puntuaciones podrían considerarse aceptables considerando el escaso número de ítems incluidos en su cálculo y que algunos autores establecen una fiabilidad mínima de .50 para propósitos de investigación (Rosenthal y Rosnow, 1991).

A continuación encontrarás una serie de afirmaciones sobre el uso del preservativo en diferentes momentos de una relación sexual. Señala tu grado de acuerdo con cada una de estas frases:

1. Me siento/sentiría cómodo/a o seguro/a a la hora de comprar preservativos

2. Me resulta/ ría cómodo hablar acerca de la utilización del preservativo con una pareja antes de comenzar la relación sexual

3. Si tengo que sugerir a una pareja que usemos el preservativo, tengo/tendría miedo de que él/ ella me rechace

4. No me siento/sentiría seguro/a a la hora de sugerir el uso del preservativo a una nueva pareja ya que él/ ella podría pensar que lo hago porque creo que tiene una ITS (Infección de Transmisión Sexual)

5. Estoy seguro/a de que recordaría utilizar el preservativo aunque haya consumido alcohol $u$ otras drogas

6. Me siento/sentiría incómodo/ a a la hora de ponerme el condón o ponérselo a mi pareja

7. Estoy seguro/a de que podría parar aún en el momento de mayor excitación para ponerme el condón o ponérselo a mi pareja

\begin{tabular}{|c|c|c|c|c|c|}
\hline 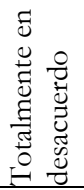 & 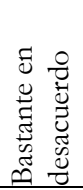 & 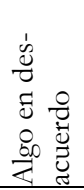 & 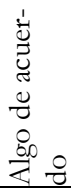 & 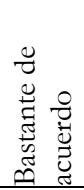 & 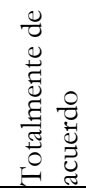 \\
\hline 0 & 1 & 2 & 3 & 4 & 5 \\
\hline 0 & 1 & 2 & 3 & 4 & 5 \\
\hline 5 & 4 & 3 & 2 & 1 & 0 \\
\hline 5 & 4 & 3 & 2 & 1 & 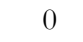 \\
\hline 0 & 1 & 2 & 3 & 4 & \\
\hline 5 & 4 & 3 & 2 & 1 & 0 \\
\hline 0 & 1 & 2 & 3 & 4 & \\
\hline
\end{tabular}

Figura 1. Subescala de autoeficacia en el uso del preservativo del CPS
Para profundizar en la relación entre la autoeficacia en el uso del preservativo y otras variables que la literatura científica ha considerado relevantes en la prevención del VIH, se emplearon ítems incluidos en los componentes del CPS: frecuencia autoinformada de uso del preservativo, intención de conducta en una situación en la que no se dispusiera de pre- servativo, actitudes y percepción de riesgo (véase Figura 2). Por otra parte, para obtener el uso autoinformado del preservativo, además de evaluarlo en función de la práctica, del tipo de pareja y del consumo de sustancias, calculamos un índice compuesto a partir del sumatorio de todos los ítems incluidos en el componente conductual.

\begin{tabular}{|c|c|c|}
\hline Componente & Ítem & Formato de respuesta \\
\hline Conducta & $\begin{array}{l}\text { ¿Con qué frecuencia has utilizado el preservativo ante las si- } \\
\text { guientes prácticas o situaciones? Coito vaginal, sexo oral, } \\
\text { coito anal, con pareja estable, con pareja esporádica } \\
\text { y bajo los efectos de alcohol/drogas }\end{array}$ & $\begin{array}{l}\text { Tipo Likert con cuatro alternativas de respuesta: } \\
\square \text { Nunca } \\
\square \text { Algunas veces } \\
\square \text { Bastantes veces } \\
\square \text { Siempre }\end{array}$ \\
\hline $\begin{array}{l}\text { Intención } \\
\text { de conducta }\end{array}$ & $\begin{array}{l}\text { ¿Qué biciste/ harias en el caso de haber mantenido alguna pa- } \\
\text { reja esporádica mientras tenías una pareja estable y no bubie- } \\
\text { ras utilizado preservativo? }\end{array}$ & $\begin{array}{l}\text { Elección múltiple con cuatro alternativas de respuesta: } \\
\square \text { Comenzar a usar el preservativo con mi pareja inven- } \\
\text { tando alguna excusa } \\
\square \text { Seguir sin utilizar el preservativo como si no hubiera } \\
\text { pasado nada } \\
\square \text { Decir la verdad y empezar a utilizar el preservativo } \\
\square \text { Decírselo y decidir no utilizar el preservativo }\end{array}$ \\
\hline $\begin{array}{l}\text { Actitudes hacia el } \\
\text { uso del } \\
\text { preservativo }\end{array}$ & $\begin{array}{l}\text { ¿Hasta qué punto consideras que el preservativo es } \\
\text { un método fiable de prevención sexual del SIDA? }\end{array}$ & $\begin{array}{l}\text { Tipo Likert con cuatro alternativas de respuesta: } \\
\square \text { Nada } \\
\square \text { Algo } \\
\square \text { Bastante } \\
\square \text { Mucho }\end{array}$ \\
\hline Percepción de riesgo & $\begin{array}{l}\text { Probabilidad percibida de infección por VTH } \\
\text { Temor a la infección por VIH }\end{array}$ & $\begin{array}{l}\text { Señala de } 0 \text { a } 100 \text { la probabilidad o el riesgo que percibes } \\
\text { de infectarte con el VIH } \\
\text { Señala de } 0 \text { a } 100 \text { el temor que te produce la posibilidad } \\
\text { de infectarte con el VIH }\end{array}$ \\
\hline
\end{tabular}

Figura 2. Descripción de los ítems utilizados del CPS ordenados por componentes. 


\section{Procedimiento}

La obtención de los datos se llevó a cabo entre los cursos académicos 2006 y 2008, en el marco de un proyecto de investigación más amplio que tiene como objetivo analizar la existencia de un posible perfil psicológico de riesgo para la infección por VIH en jóvenes universitarios. Los sujetos participaron en las mesas divulgativas creadas con motivo del Día Mundial de la Lucha contra el Sida por la Unidad de Investigación Sobre Sexualidad y Sida de la Universidad Jaume I de Castellón, para sensibilizar y ofrecer a los estudiantes información al respecto. El cuestionario fue cumplimentado voluntariamente, de forma individual y anónima, de manera que se garantizara completamente la confidencialidad de los datos recogidos.

\section{Análisis de datos}

Los análisis estadísticos se realizaron con el paquete estadístico SPSS-17. Tras los análisis descriptivos para la muestra total se utilizó la prueba estadística paramétrica t de student para determinar la presencia de diferencias significativas en autoeficacia en función del género de los participantes. Asimismo, se realizó un análisis de correlación de Pearson con el índice compuesto sobre frecuencia del uso del preservativo y Rho de Spearman, para examinar cómo está relacionada la autoeficacia y diferentes variables cuya respuesta sigue una escala tipo Likert, como por ejemplo la frecuencia de uso del preservativo en diferentes prácticas y la confianza en la utilización del preservativo; una escala de razón, como por ejemplo la probabilidad y el temor percibido a una posible infección; o dicotómica con cada una de las alternativas del ítem referido a la intención de conducta.

\section{Resultados}

\section{Autoeficacia en el uso del preservativo en la muestra general}

La mayor parte de la muestra analizada $(67.9 \%)$ presenta niveles medio-altos en la puntuación de autoeficacia en el uso del preservativo, con una puntuación media de 26.57 $(D T=5.26)$ sobre un total de 35 puntos obtenidos en esta escala. Teniendo en cuenta que hemos considerado tres posibles rangos de puntuaciones a efectos de su interpretación (autoeficacia baja, de 0 a 12 puntos; media, de 13 a 24 puntos; y alta de 25 a 35 puntos), un $0.7 \%$ de los participantes presenta niveles bajos y un $25.1 \%$ niveles medios. Sin embargo, resulta interesante comparar las diferencias existentes entre los ítems que se refieren a los diferentes momentos de una relación sexual. Como podemos comprobar en la Tabla 1, se observa que la muestra evaluada se siente más autoeficaz si tiene que sugerir el uso del preservativo sin considerar que por eso la pareja podría rechazarles y a la hora de sugerirlo a una nueva pareja porque pudiera pensar que lo hace creyendo que él/ella tiene alguna infección de transmisión sexual (ITS). Por otra parte, encontramos que a los jóvenes universitarios de la muestra les resulta más cómodo hablar de la utilización del preservativo que ponérselos en la relación sexual o comprarlos. Por último, parecen mostrar menor autoeficacia en lo que respecta a la seguridad de poder parar en el momento de mayor excitación.

\section{Autoeficacia en el uso del preservativo: diferencias de género}

Analizando las diferencias que surgen en función del género de la muestra, son las mujeres las que obtienen una puntuación superior en la escala global de autoeficacia, respecto a los hombres, siendo las diferencias estadísticamente significativas mediante una Prueba t. No obstante, es importante comparar las diferencias existentes en cuanto a las expectativas que tienen ambos sexos para poner en práctica las habilidades necesarias a la hora de utilizar el preservativo en distintos momentos de la relación sexual. En general, las mujeres presentan mayores puntuaciones medias que los hombres en todas las situaciones analizadas, excepto en la comodidad o seguridad a la hora de comprar el preservativo, ítem en el cual los hombres obtienen una media superior a las mujeres. Ocurre lo contrario cuando hacemos referencia al ámbito privado de una relación, es decir, las mujeres sienten mayor comodidad tanto en el momento de poner el preservativo como a la hora de hablar de su utilización antes de comenzar una relación sexual. En ambos grupos, las situaciones ante las que se perciben más autoeficaces, son aquellas que se refieren a una posible evaluación negativa o rechazo por parte de la pareja cuando se sugiere o propone el uso del preservativo. Sin embargo, el ítem que genera menor autoeficacia en ambos sexos es la seguridad de parar en el momento de máxima excitación para ponerse el preservativo o ponérselo a la pareja. Cabe destacar que es en esta situación donde mayores son las diferencias entre hombres y mujeres, siendo ellos los que se perciben mucho menos autoeficaces. Cuando realizamos una Prueba t con cada uno de los ítems de la escala, obtenemos en todos ellos diferencias estadísticamente significativas en función del género (véase Tabla 1).

\section{Relación entre autoeficacia y el uso del preservativo en distintas prácticas sexuales}

La utilización del preservativo es la principal medida de prevención frente al VIH. Por ello calculamos la correlación de la puntuación global de autoeficacia con la frecuencia total de uso del preservativo no encontrándose relaciones significativas a nivel estadístico $(r=.041, p \leq .341)$. Si atendemos al tipo de práctica, encontramos correlaciones estadísticamente significativas con signo positivo en el coito vaginal (Rho $=.077, p \leq .000$ ), aunque como vemos con un valor menor a .10. Es decir, aquellas personas que se perciben más autoeficaces a la hora de usar el preservativo tienden a utilizarlo más frecuentemente en coito vaginal. Las correlaciones 
no fueron significativas en el sexo anal (Rho=.052, $p \leq .151)$ ni oral (Rho $=-.034, p \leq .122)$, probablemente como consecuencia de la escasa prevalencia de la primera práctica y de la escasa frecuencia del uso del preservativo en la segunda. En función del tipo de pareja obtuvimos correlaciones significativas a nivel estadístico, también con valores bajos, con el uso del preservativo en el marco de la pareja estable
(Rho $=.068, p \leq .001)$ y en relaciones con parejas esporádicas (Rho $=.171, p \leq .000)$. Por otro lado, también se encontraron en relaciones que se mantienen bajo los efectos del alcohol o las drogas (Rho=.170, $p \leq .000)$. Por lo tanto, mayores puntuaciones en autoeficacia global significarían un mayor uso del preservativo en las situaciones descritas anteriormente.

Tabla 1. Diferencias de género en la subescala de autoeficacia del CPS

\begin{tabular}{|c|c|c|c|c|c|c|c|}
\hline \multirow[b]{2}{*}{ Variable: } & \multicolumn{2}{|c|}{$\begin{array}{l}\text { Muestra total } \\
(N=3530)\end{array}$} & \multicolumn{2}{|c|}{$\begin{array}{l}\text { Hombres } \\
(N=1732)\end{array}$} & \multicolumn{2}{|c|}{$\begin{array}{l}\text { Mujeres } \\
(N=1798)\end{array}$} & \multirow[b]{2}{*}{$t$} \\
\hline & $M$ & $D T$ & $M$ & $D T$ & $M$ & $D T$ & \\
\hline Autoeficacia general & 26.57 & 5.26 & 25.49 & 5.41 & 27.59 & 4.91 & $-11.751 * * *$ \\
\hline 1. Me siento/sentiría cómodo/a o seguro/a a la hora de comprar preservativos & 3.68 & 1.47 & 3.81 & 1.43 & 3.56 & 1.50 & $4.916 * * *$ \\
\hline $\begin{array}{l}\text { 2. Me resulta/ ría cómodo hablar acerca de la utilización del preservativo con una pareja } \\
\text { antes de comenzar la relación sexual }\end{array}$ & 4.00 & 1.24 & 3.89 & 1.28 & 4.10 & 1.20 & $-4.873 * * *$ \\
\hline $\begin{array}{l}\text { 3. Si tengo que sugerir a una pareja que usemos el preservativo, tengo/tendría miedo de } \\
\text { que él/ ella me rechace }\end{array}$ & 4.22 & 1.28 & 4.05 & 1.37 & 4.38 & 1.17 & $-7.466 * * *$ \\
\hline $\begin{array}{l}\text { 4. No me siento/sentiría seguro/a a la hora de sugerir el uso del preservativo a una nueva } \\
\text { pareja ya que él/ ella podría pensar que lo hago porque creo que tiene una ITS }\end{array}$ & 4.21 & 1.36 & 4.01 & 1.47 & 4.40 & 1.23 & $-8.512 * * *$ \\
\hline $\begin{array}{l}\text { 5. Estoy seguro/ a de que recordaría utilizar el preservativo aunque haya consumido alco- } \\
\text { bol } u \text { otras drogas }\end{array}$ & 3.73 & 1.40 & 3.59 & 1.47 & 3.87 & 1.31 & $-5.992 * * *$ \\
\hline 6. Me siento/sentiria incómodo/a a la hora de ponerme el condón o ponérselo a mi pareja & 3.69 & 1.64 & 3.49 & 1.69 & 3.90 & 1.56 & $-7.511 * * *$ \\
\hline $\begin{array}{l}\text { 7. Estoy seguro/ a de que podría parar aún en el momento de mayor excitación para po- } \\
\text { nerme el condón o ponérselo a mi pareja }\end{array}$ & 2.98 & 1.74 & 2.60 & 1.77 & 3.35 & 1.63 & $-12.806 * * *$ \\
\hline
\end{tabular}
póérselo a mi pareja Nota: $* * *: p \leq .000$

\section{Relación entre autoeficacia y otras variables relacio- nadas con la prevención del VIH}

Por último, examinamos si la autoeficacia global en el uso del preservativo se relacionaba con otras variables que se han mostrado relevantes en el ámbito de la prevención del VIH: la intención de conducta, la percepción de riesgo y la confianza hacia el preservativo. En primer lugar, se analizaron las correlaciones existentes entre la puntuación global de autoeficacia y cuatro alternativas de respuesta referidas a la intención de conducta ante la posibilidad de mantener relaciones sexuales sin preservativo con alguna pareja esporádica mientras se tiene una pareja estable. En este sentido obtenemos correlaciones estadísticamente significativas con signo positivo aunque con valores inferiores a .10 en aquellas conductas que resultarían más preventivas: no mantener relaciones sexuales (Rho $=.040, p \leq .030) \mathrm{e}$ ir en busca de un preservativo $(\mathrm{Rho}=.083, p \leq .000)$. Por el contrario, encontramos correlaciones con signo negativo en el comportamiento que implica máximo riesgo, es decir, tener la relación de igual forma (Rho $=-.136, p \leq .000)$. La correlación con la alternativa de tener la relación evitando prácticas de riesgo (Rho=-.016, $p \leq .398$ ) no alcanza la significación estadística. Por otro lado, analizamos otros aspectos relacionados con la variable percepción de riesgo. Con respecto a la probabilidad percibida de infectarse por VIH, obtenemos correlaciones estadísticamente significativas con signo negativo (Rho=$.119, p \leq .000)$. Mientras que el temor percibido correlaciona de forma significativa con signo positivo (Rho=.052, $p \leq .005)$. Finalmente, observamos correlaciones significativas con signo positivo entre autoeficacia y confianza en el pre- servativo como método fiable de prevención del VIH (Rho=.146, $p \leq .000)$.

\section{Discusión y conclusiones}

La muestra evaluada en nuestro estudio ha obtenido niveles medio-altos en la escala utilizada para evaluar la autoeficacia en el uso del preservativo. Sin embargo, varían en función del tipo de comportamiento considerado, presentando mayor seguridad en aquellos aspectos relacionados con una posible evaluación negativa por parte de la pareja cuando se sugiere su uso, y menor autoeficacia en aquellas situaciones referidas al contexto público o que impliquen el control de la conducta en el mismo momento de la relación sexual. En función del género, en la línea de otros estudios como el de Burns y Dillon (2005) o Parsons et al. (2000), la evaluación de la autoeficacia nos sugiere que son las mujeres las que expresan sentirse más capaces en general, a la hora de resolver situaciones relacionadas con la utilización del preservativo en diferentes momentos de la relación sexual. Sin embargo, Muñoz-Silva et al. (2009) obtuvieron resultados contrarios con población española y portuguesa, siendo los hombres los que se sentían más autoeficaces a la hora de utilizar los preservativos, incluso en situaciones que consideraban difíciles. Si atendemos al análisis de las situaciones planteadas en nuestro estudio cabe mencionar algunos aspectos relevantes. En primer lugar, tanto en el caso de los hombres como de las mujeres, las situaciones en las que se perciben más autoeficaces, son aquéllas que describen un posible rechazo o evaluación negativa por parte de la pareja. Por el contrario, 
es la seguridad de parar para usar el preservativo, en el momento de máxima excitación, en la que los jóvenes universitarios se consideran menos autoeficaces. Parece pues que los jóvenes de nuestro estudio consideran que poseen las habilidades necesarias para afrontar situaciones que se dan antes de que se inicie la relación sexual, aunque conlleven el componente emocional del miedo o temor a una evaluación social negativa frente a la sugerencia del uso del preservativo. $\mathrm{Y}$ sin embargo, carecen de las habilidades de autocontrol emocional y conductual cuando nos trasladamos al momento preciso de la interacción sexual, sobretodo en el caso de los hombres. Por otra parte, la única situación en la que éstos puntúan más alto que las mujeres se refiere a la comodidad a la hora de comprar preservativos. A pesar que en la actualidad las diferencias en los roles sexuales de hombres y mujeres se han ido transformando, este resultado nos demuestra, la mayor seguridad de los hombres para manejar una situación pública relacionada con la sexualidad y el uso del preservativo, y por el contrario la mayor autoeficacia percibida por parte de las mujeres para manejar este tema en el ámbito privado. Por ejemplo, sintiéndose más seguras a la hora de hablar sobre su utilización como demuestra el metanálisis realizado por Allen et al. (2002). Como sugiere Quatrella (2000) esto es debido, quizá, a la mayor responsabilidad que se les atribuye socialmente a los hombres en este aspecto o a la experiencia de emociones negativas, como vergüenza, por parte de las mujeres.

Por otra parte, como nos han mostrado las correlaciones, la autoeficacia se relaciona positivamente con la frecuencia del uso del preservativo en distintas prácticas sexuales (coito vaginal, pareja estable, pareja esporádica y bajo efectos de alcohol o drogas), sobre todo en el caso del coito vaginal coincidiendo con los resultados obtenidos por Matsuura (2009), y con la intención de realizar una conducta preventiva. En esta línea se sitúan también los trabajos de Dilorio et al. (2000), Mashegoane et al. (2004), Ozakinci y Weinman (2006) o Svenson et al. (2002). Por otra parte, parece lógico pensar que las personas que se consideran más capaces de utilizar el preservativo en sus relaciones sexuales son aquellas que mantienen una actitud más favorable hacia este método

\section{Referencias}

Allen, M, Emmers-Sommer, T. y Crowell, T. (2002). Couples negotiating safer sex behaviors: a meta-analysis of the impact of conversation and sex. En M. Allen, R. Preiss, B. Gayle y N. Burrell (Eds.), Interpersonal communication research: advances through meta-analysis (pp. 263-280). Mahwah, NJ: Lawrence Erlbaum.

Ballester, R., Gil, MD. y Giménez, C. (2007, Junio). El "Cuestionario de Prevención del Sida (CPS): Análisis de la fiabilidad y validez" (pp.135). Investigación presentada en el X Congreso Nacional sobre el Sida de la Sociedad Española Interdisciplinaria de Sida, San Sebastián, España.

Ballester, R., Gil, M.D., Giménez, C. y Ruiz, E. (2009). Actitudes y conductas sexuales de riesgo para la Infección por VIH/Sida en jóvenes españoles. Revista de Psicopatología y Psicología Clínica, 14(3), 181-191.

Bandura, A. (1994). Social Cognitive Theory and exercise of control over HIV infection. En R. J. DiClemente y J. L. Peterson (Eds.), Preventing AIDS: Theories and methods of behavioral intervention (cap. 3). Nueva York: Plenum Press. como medida preventiva y, por lo tanto, se perciban menos vulnerables a la infección por VIH.

Así pues, consideramos que la autoeficacia en el ámbito del VIH puede contribuir a desarrollar conductas seguras y a mantener la motivación necesaria para una constante prevención, evitando de esta forma la infección por VIH. Aspectos tales como la escasa familiaridad con el uso del preservativo, la excitación que acompaña la relación sexual, la falta de habilidades de comunicación en la pareja, la escasa capacidad de autocontrol y la falta de asertividad para la negociación del sexo seguro pueden suponer barreras importantes para la prevención del VIH. Tal como sugieren Robles et al. (2006), en las intervenciones preventivas se deberían trabajar las habilidades conductuales relacionadas con el comportamiento sexual preventivo, y no sólo el aspecto informacional de la prevención del VIH. Resulta importante reflexionar acerca de la necesidad de incluir un componente de entrenamiento en autoeficacia en las futuras intervenciones preventivas, de forma que se trabaje la adquisición o fortalecimiento de aquellas actitudes y habilidades necesarias para el uso correcto del preservativo antes y durante la relación sexual. Del mismo modo, atender los factores situacionales y el tipo de pareja (Planes, 1995) y preparar en el afrontamiento de situaciones consideradas complicadas o difíciles, como por ejemplo el control de los impulsos sexuales, que pueden dar lugar a conductas sexuales de riesgo. No obstante, creemos que en la sociedad subyacen creencias y valores respecto a la sexualidad y a la responsabilidad del uso del preservativo, diferentes en función del género. De igual forma a lo que sugieren otros estudios (Allen et al., 2002; Matsuura, 2009, Muñoz-Silva et al., 2009), no cabe duda que las intervenciones diseñadas deberían incluir una perspectiva de género, para obtener resultados más efectivos y adaptados a las necesidades del colectivo con el que estamos trabajando.

Agradecimientos.- Proyecto financiado por la Universitat Jaume I-Fundació Bancaixa (P1 1B2006-19) y Fundación para la Investigación y la Prevención del Sida en España (exp. 36639/07).

Bayés, R. (1995). Sida y Psicologia. Madrid: Martínez Roca.

Burns, M. J. y Dillon, F. R. (2005). AIDS health locus of control, selfefficacy for safer sexual practices and future time orientation as predictors of condom use in African American college students. Journal of Black Psychology, 31(2), 172-188.

Casey, M. K., Timmerman, L., Allen, M, Krahn, S. y LaPlant, K. (2009). Response and self-efficacy of condom use: a meta-analysis of this important element of AIDS education and prevention. Southern Communication Journal, 74(1), 57-78.

Dilorio, C., Dudley, W. N., Soet, J., Watkins, J. y Maibach, E. (2000). A social cognitive-based model for condom use among college students. Nursing Research, 49(4), 208-214.

Erlen, J. A., Cha, E. S., Kim, K. H., Caruthers, D. y Sereika, S. M. (2010) The HIV Medication Taking Self-efficacy Scale: psychometric evaluation. Journal of Advanced Nursing, 66(11), 2560-2572. 
Espada, J. P. y Quiles, M. J. (2001). Prevenir el sida. Guía para padres y educadores. Madrid: Pirámide.

Farmer, M. A. y Meston, C. M. (2006). Predictors of condom use selfefficacy in an ethnically diverse university sample. Archives of Sexual Behavior, 35(3), 313-326.

Gómez, S., Ballester, R., Gil, MD., Gil, B. y Salmerón, P. (2009, Mayo). Propiedades psicométricas de una escala de autoeficacia para la prevención del Sida en jóvenes universitarios. Investigación presentada en el XII Congreso Nacional sobre el Sida de la Sociedad Española Interdisciplinaria de Sida, Valencia, España.

Lameiras, M., Faílde, J. M., Saco, A. y Rodríguez, Y. (2006). A qualitative study of the viability of usage of the female condom among university students. International Journal of Clinical and Health Psychology, 6(001), 189199.

Lindemann, D. F. y Brigham, T. A. (2003). A Guttman Scale for assessing condom use skills among college students. AIDS and Behavior, 7(1), 2327.

Mashegoane, S., Moalusi, K. P., Ngoepe, M. A. y Peltzer, K. (2004). The prediction of condom use intention among South African university students. Psychological Reports, 95(2), 407-417.

Matsuura, A. C. (2009). Interpersonal and psychosexual factors predicting HIV-related risky sex behaviors in heterosexually active women and men. Dissertation Abstracts International: Section B: The Sciences and Engineering, 69(9-B), 5833.

Muñoz-Silva, A., Sánchez-García, M., Martins, A. y Nunes, C. (2009). Gender differences in HIV-related sexual behavior among college students from Spain and Portugal. The Spanish Journal of Psychology, 12(2), 485-495.

O'Leary, A, Jemmott, L. y Jemmot III, J. B. (2008). Mediation analysis of an effective sexual risk-reduction intervention for women: the importance of self-efficacy. Health Psychology, 27(2), S180-S184.

Ozakinci, G. y Weinman, J. A. (2006). Determinants of condom use intentions and behavior among Turkish Youth. Journal of HIV/AIDS Prevention in Children and Youth, 7(1), 73-95.

Parsons, J. T., Halkitis, P. N., Bimbi, D. y Borkowski, T. (2000). Perceptions of the benefits and costs associated with condom use and unprotected sex among late adolescent college students. Journal of Adolescence, 23, 377-391.

Planes, M. (1995). Expectativas de autoeficacia y su relación con los comportamientos sexuales preventivos frente al Sida. Avances en Psicología Clínica Latinoamericana, 13, 27-37.
Planes, M., Gras, M. E., Soto, J. y Font-Mayolas, S. (2000). Percepción de riesgo y comportamientos heterosexuales relacionados con la prevención del sida en jóvenes universitarios. Análisis y Modificación de Conducta, 26(107), 265-389.

Programa de Naciones Unidas para el Desarrollo (2006). Una alianza mundial para el desarrollo. Recuperado el 17 de Enero de 2011, de http://www.undp.org/spanish/publicaciones/informeanual2006/IAR0 6_SP.pdf

Quatrella, L. A. (2000). Predictors of condom use self-efficacy and perceptions of responsabilities of safer sex behaviors among college students. Dissertation Abstracts International: Section B: The Sciences and Engineering, 61(4-B), 2275.

Robles, S., Moreno, D., Frías, B., Rodríguez, M., Barroso, R., Díaz, E. y Hernández R. (2006). Entrenamiento conductual en habilidades de comunicación sexual en la pareja y uso correcto del condón. Anales de Psicología, 22(1), 60-71.

Rosenthal, R. y Rosnow, R. L. (1991). Essential of behavioral research, methods and data analysis. Boston: McGraw-Hill.

Secretaría del Plan Nacional sobre Sida del Ministerio de Sanidad y Política Social de España (2009). Vigilancia epidemiológica del sida en España: registro nacional de casos de sida. Informe semestral $n^{\circ} 2$. Recuperado el 17 de Enero de 2011, de

http://www.iscii.es/htdocs/epidemiologia/pdf/SPNS_Informe_semes tral.pdf

Svenson, G. R., Oestergren, P., Merlo, J. y Rastam, L. (2002). Action control and situational risks in the prevention of HIV and STIs: individual, dyadic and social influences on consistent condom use in a university population. AIDS Education and Prevention, 14, 515-531.

UNAIDS (2010). Unaids report on the global AIDS epidemic. Recuperado el 17 de Enero de 2011, de http://www.unaids.org/documents/20101123_GlobalReport_em.pdf

Weeks, K., Levy, S. R., Zhu, Ch., Perhats, C. Handler, A. y Flay, B. R. (1995). Impact of a social-based AIDS prevention program on young adolescents' self-efficacy skills. Health Education Research, 10(3), 329-344.

Wulfert, E. y Wan, Ch. K. (1993). Condom use: a Self-efficacy Model. Healt Psychology, 12(3), 346-353.

(Artículo recibido: 31-03-2011, revisión: 11-01-2012, aceptado: 20-01-2012) 